\title{
Critical Dose of Lead Affecting $\delta$-Aminolevulinic Acid Levels
}

\author{
Katsuyuki Murata ${ }^{1}$, Tadashi SAKAI ${ }^{2}$, Yoko Morita ${ }^{2}$, Toyoto IwATA ${ }^{1}$ and Miwako DakeIshi ${ }^{1}$ \\ ${ }^{1}$ Environmental Health Sciences, Department of Social Medicine, Akita University School of Medicine and \\ ${ }^{2}$ Occupational Poisoning Center, Tokyo Labor Accident Hospital, Japan
}

\begin{abstract}
Critical Dose of Lead Affecting $\delta$ Aminolevulinic Acid Levels: Katsuyuki Murata, et al. Akita University School of Medicine-To estimate the critical dose of the association between the blood lead concentration $(\mathrm{BPb})$ and $\delta$-aminolevulinic acid (ALA) levels, ALA levels in plasma (ALA-P), blood (ALA$B)$, and urine (ALA-U), and the activity of $\delta$ aminolevulinic acid dehydratase (ALAD) were determined in 186 Japanese lead workers, aged 18$62 \mathrm{yr}$, with BPb levels of $2.1-62.9 \mu \mathrm{g} / \mathrm{d} /$. For this purpose, the benchmark dose (BMD) method, recently used in the environmental health field in place of the no-observed-adverse-effect level, was introduced into this study. The BMD was defined as the BPb level that resulted in an increased probability of abnormal change in ALA-related parameters by an excess risk (BMR) of $5 \%$ in exposed workers i.e., from $P_{0}$ (abnormal probability of $5 \%$ in unexposed workers) to $P_{0}+B M R$ for exposed workers at the BMD. ALA-related parameters were significantly correlated with $\mathrm{BPb}$. The BMDs computed from the 186 workers, after controlling for age, were 15.3-20.9 $\mu \mathrm{g} / \mathrm{d} /$ for ALA levels, and 2.9 $\mu \mathrm{g} / \mathrm{d} /$ for ALAD; likewise, the BMDs from the 154 workers with $\mathrm{BPb}$ levels of less than $40 \mu \mathrm{g} / \mathrm{d} /$ were 3.3$8.8 \mu \mathrm{g} / \mathrm{d} /$ for ALA levels, and $2.7 \mu \mathrm{g} / \mathrm{d} /$ for ALAD. Since the cutoff value of ALA-P, computed from the latter workers, seems to be closer to the upper normal limit in unexposed adults than does that from the former workers, it is suggested that the critical dose of $\mathrm{BPb}$ causing the increased levels of ALA is below $10 \mu \mathrm{g} / \mathrm{d} /$. Such critical doses are necessary to promote preventive activities of adverse effects of lead. (J Occup Health 2003; 45: 209-214)
\end{abstract}

Key words: Lead, Critical dose, Adverse effect, $\delta$ Aminolevulinic acid, Occupational exposure, $\delta$ Aminolevulinic acid dehydratase

The immediate effect of the inhibition of $\delta$ -

Received Jan 20, 2003; Accepted April 30, 2003

Correspondence to: K. Murata, Environmental Health Sciences, Department of Social Medicine, Akita University School of Medicine, 1-1-1 Hondo, Akita 010-8543, Japan aminolevulinic acid dehydratase (ALAD) due to lead exposure is an increased level of $\delta$-aminolevulinic acid (ALA) in the plasma (ALA-P) and blood (ALA-B), which then leads to increased urinary excretion (ALA-U) ${ }^{1,2}$. Selander and $\mathrm{Cramer}^{3)}$ showed a clear threshold effect at about $40 \mu \mathrm{g} / \mathrm{d} l$ in occupational subjects, regarding the correlation between the blood lead concentration $(\mathrm{BPb})$ and ALA-U. Based upon several studies on the associations between $\mathrm{BPb}$ and ALA levels ${ }^{3-6)}$, lead has been suggested to have discernible effects on the ALA$\mathrm{U}$ at a $\mathrm{BPb}$ level of around $35 \mu \mathrm{g} / \mathrm{d} l^{1}$. Likewise, the threshold of $\mathrm{BPb}$ for decreased ALAD activity has been estimated to be approximately $10 \mu \mathrm{g} / \mathrm{d} l^{1}$. However, since these values may differ according to the used data (e.g., those including an inadequate population) or the mathematical model used for the estimation ${ }^{7)}$, they should be reconfirmed by using the latest technique; because, an allowable exposure dose computed from such values has been used as a measure of the "acceptability" of occupational exposure levels.

The no-observed-adverse-effect level (NOAEL) is the highest dose at which no statistically or biologically significant adverse effects are identified ${ }^{8)}$. On the other hand, the benchmark procedure is a statistical tool used to determine an allowable exposure to a toxic chemical ${ }^{9,10)}$. The benchmark dose (BMD) is a dose that causes a prescribed adverse change in response. Recently, the BMD has been used in the field of environmental health in place of the NOAEL ${ }^{11-14)}$, because many shortcomings of the NOAEL have been identified, including not adequately reflecting the shape of the dose response and not appropriately accounting for study size ${ }^{8,10)}$.

Lead at low levels of exposure has been recognized to be toxic ${ }^{15}$, and it would be important to discern a threshold for lead toxicity in populations exposed to lead. For this reason, the benchmark procedure was introduced into this study to identify the $\mathrm{BPb}$ level at which abnormal changes in ALA-related parameters emerged in lead workers.

\section{Methods}

At the time of the special health checkup for lead 
workers, conducted under the Industrial Safety and Health Law in Japan, the nature of the procedure used in the present study was fully explained to all workers, and the study was carried out with their informed consent. Venous blood treated with heparin or EDTA-2K was taken from 186 male lead workers (18-62 yr old, mean 43). The lead workers were employed in a secondary smelter, in a glass factory, and in electrical appliance manufacture (soldering). Plasma was separated from whole blood immediately after sampling, stored at $4^{\circ} \mathrm{C}$ or $-80^{\circ} \mathrm{C}$, and used for the determination of ALA-P. Spot urine was also collected from the lead workers, and stored at $-20^{\circ} \mathrm{C}$ until analysis.

\section{Measurement of ALA-related parameters}

ALA-P and ALA-B were determined by the methods previously reported ${ }^{16-18)}$. Briefly, $40 \mu \mathrm{l}$ of $25 \%$ trichloroacetic acid (TCA) and $10 \mu l$ of $30 \mathrm{mM}$ iodoacetamide were added to $100 \mu l$ of plasma or blood, and vigorously agitated with a vortex mixer. To determine ALA-P, iodoacetamide could be replaced by distilled water. After centrifugation at $13,000 \mathrm{rpm}$ for $10 \mathrm{~min}$ in a microcentrifuge, $10 \mu l$ of the supernatant was used for the derivatization. The standard reaction mixture for the derivatization contained $10 \mu \mathrm{l}$ of the supernatant, $240 \mu \mathrm{l}$ of distilled water, $250 \mu l$ of $200 \mathrm{mM}$ acetate buffer ( $\mathrm{pH}$ 3.8), $1.25 \mathrm{~m} l$ of solution A (acetylacetone-ethanol-water 15: $10: 55 \mathrm{v} / \mathrm{v} / \mathrm{v})$, and $250 \mu l$ of solution B $(8.5 \% \mathrm{w} / \mathrm{v}$ formaldehyde solution). The reaction was carried out at boiling point for $15 \mathrm{~min}$. After cooling, the mixture was used for the HPLC analyses.

A liquid chromatograph (Shimadzu, Kyoto, Japan) consisting of a pump (LC-10A), an automatic sample injector (SIL-10A), a column oven (CTO-10A), a fluorescence detector (RF-550A) and a data processor (C-R4A) was used. The column $(150 \times 6 \mathrm{~mm})$ was packed with reversed phase silica (Inertsil ODS-2, GL Science, Tokyo, Japan). The mobile phase was $50 \%$ methanol containing $0.1 \%$ acetic acid. The flow rate, oven temperature, and detector wavelength were set at $0.7 \mathrm{ml} / \mathrm{min}, 40^{\circ} \mathrm{C}$, and $373 \mathrm{~nm} / 463 \mathrm{~nm}$ (excitation/ emission), respectively. Samples were cooled at $4{ }^{\circ} \mathrm{C}$ during a series of analyses and $80 \mu l$ was automatically injected at 20-min intervals.

The method of ALA-U determination was basically the same as that used by Okayama et al. ${ }^{19)}$ ALA-U was corrected for the creatinine concentration (ALA-U, mg/g Cre). Creatinine was determined by the method of Jaffe with the "Creatinine Determination Kit" (Wako Pure Chemicals, Tokyo, Japan). Erythrocyte ALAD activity was determined by the Commission of European Communities (CEC) standard method, and the activity was expressed as units ( $\mathrm{u}: \mu \mathrm{mol}$ ALA/min/l RBC) ${ }^{20)}$. $\mathrm{BPb}$ was determined by flameless atomic absorption spectrometry (Hitachi Z-8000, Tokyo, Japan).

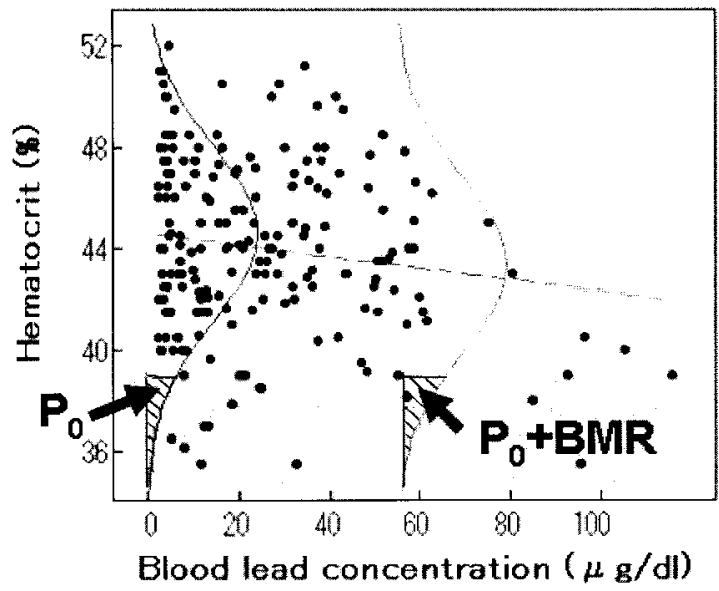

Fig. 1. Dose-effect relation of the blood lead concentration to hematocrit in lead workers for benchmark dose (BMD) calculation. $\mathrm{P}_{0}$ and benchmark response (BMR) indicated an abnormal probability $(5 \%)$ in unexposed workers and an excess risk $(5 \%)$ above $\mathrm{P}_{0}$ in exposed workers, respectively. The power parameter of $\mathrm{K}=1$ was used in this figure to simplify the model.

\section{Statistical analysis}

The BMD was defined as the BPb level that resulted in an increased probability of abnormal change in ALArelated parameters by a benchmark response (BMR), i.e., from $\mathrm{P}_{0}$ for unexposed subjects to $\mathrm{P}_{0}+\mathrm{BMR}$ for exposed subjects at the BMD (Fig. 1) ${ }^{9,10}$. Previous applications of this method have used a $\mathrm{P}_{0}$ (i.e., an abnormal probability in outcome data of unexposed subjects) of $5 \%$ and a BMR (i.e., an excess risk in exposed subjects) of $5 \% 8,13,14)$. Although observational studies have not included an unexposed group completely free of exposure, data of the group could be extrapolated from those of exposed subjects ${ }^{13)}$. And, the BMD and the cutoff value (C) of $\mathrm{P}_{0}$ were calculated by using a statistical doseresponse model based upon power functions for the dependence of ALA-related parameters on $\mathrm{BPb}(\mathrm{d})$ and the confounder: (1) $\mu$ (d) $=\beta_{0}+\beta_{1} \cdot \mathrm{d}^{\mathrm{K}}+\beta_{2} \cdot$ [age ], (2) $\mathrm{P}_{0}=\Phi$ $\left(\left(\mathrm{C}-\beta_{0}\right) / \sigma\right)$, and (3) BMD $=\left[\Phi^{-1}\left(\mathrm{P}_{0}\right)-\Phi^{-1}\left(\mathrm{P}_{0}+\mathrm{BMR}\right)\right] \cdot \sigma /$ $\beta_{1}$ ( $\Phi$ and $\sigma$ indicate the standard cumulative normal distribution function and standard deviation of ALArelated parameters in unexposed subjects, respectively). A lower confidence limit (BMDL) for BMD was then calculated as the statistical $95 \%$ lower bound of the $\mathrm{BMD}^{13)}$. The power parameter $\mathrm{K}$ has been restricted to values equal to or above 1 , thus allowing the doseresponse curve to be nonlinear ${ }^{14)}$. We applied the K-power model in accordance with recent applications ${ }^{13,14)}$. All analyses were performed by using the Statistical Package for the Biosciences (SPBS V9.5) with the BMD $\operatorname{program}^{21)}$. 


\section{Results}

Table 1 summarizes the BPb, ALA-P, ALA-B, ALA$\mathrm{U}$, and $\mathrm{ALAD}$ of the lead workers. The $\mathrm{BPb}$ level ranged from 2.1 to $62.9 \mu \mathrm{g} / \mathrm{d} l$. Since there was a negative exponential relationship between $\mathrm{BPb}$ and ALAD in the lead workers, the latter was logarithmically transformed when it was used as a dependent variable of the regression model. These ALA-related parameters were significantly correlated with $\mathrm{BPb}$ (Table 2). The BMDs for ALA levels, computed after controlling for age, were between 15.3 and $20.9 \mu \mathrm{g} / \mathrm{d} l$ (Table 3 and Fig. 2); ALA-U showed the highest BMD value among them. Also, the relation of $\mathrm{BPb}$ to log-transformed ALAD seemed to be almost linear (Fig. 2), and the BMD for log-transformed ALAD was $2.9 \mu \mathrm{g} / \mathrm{d} l$ (Table 3 ). In addition, according to the report by Sakai and Morita ${ }^{16)}$, when BMDs were recalculated in 154 lead workers who had BPb levels of less than $40 \mu \mathrm{g} /$ $\mathrm{d} l$, those for ALA levels were between 3.3 and $8.8 \mu \mathrm{g} / \mathrm{d} l$ (Table 3).

\section{Discussion}

The purpose of the current study is the estimation of the "critical dose" of lead exposure causing increased levels of ALA. If we use a $\mathrm{P}_{0}$ of $5 \%$ and a BMR of $5 \%$ in BMD calculations, the cutoff value represents the upper limit (i.e., $\mathrm{P}_{0}=0.05$ ) of a $90 \%$ confidence interval of ALA in unexposed subjects; and, since the proportion of exposed subjects with ALA levels above the cutoff value increases with elevated levels of lead, the BMD is the $\mathrm{BPb}$ level at which $10 \%$ of the exposed workers have such an abnormal ALA level on the dose-response curve. By using the K-power model, the critical dose for ALA levels was estimated to be $15.3-20.9 \mu \mathrm{g} / \mathrm{d} l$ in all subjects, and this value almost corresponded with the threshold for ALA-U estimated by Higashikawa et al. ${ }^{7)}$ It is therefore suggested that the BMD method provides a promising approach for estimating the dose-response association with hazardous factors in the field of occupational health.

On the other hand, the cutoff value of ALA-P, specified from the 154 lead workers with BPb levels below $40 \mu \mathrm{g} /$ $\mathrm{d} l$ by the BMD method, was $11.3 \mu \mathrm{g} / l$ (Table 3 ). Morita et $a l .{ }^{22)}$ reported a $95 \%$ confidence interval of 6.0-12.5 $\mu \mathrm{g} / l$ as a reference value for ALA-P; similarly, the ALA$\mathrm{P}$ level in 33 workers with BPb levels of 2.5-4.9 $\mu \mathrm{g} / \mathrm{d} l$ ranged from 6.4 to $10.4 \mu \mathrm{g} / l$ in another study ${ }^{16)}$. In comparison, the cutoff value at $\mathrm{BPb}$ levels below $40 \mu \mathrm{g}$ / $\mathrm{d} l$ seem to be closer to the upper normal limits of ALA-P than does that at BPb levels of 2.1-62.9 $\mu \mathrm{g} / \mathrm{d} l$. Moreover, when $\mathrm{BPb}$ exceeds approximately $40 \mu \mathrm{g} / \mathrm{d} l$, ALA levels have been reported to be acceleratedly elevated $\left.{ }^{7}, 16\right)$,

Table 1. Blood lead concentration (BPb), $\delta$-aminolevulinic acid levels in plasma (ALA-P), blood (ALA-B), and urine (ALA-U), and $\delta$-aminolevulinic acid dehydratase (ALAD) activity in 186 Japanese lead workers

\begin{tabular}{lcc}
\hline & Median & Range* $^{*}$ \\
\hline $\mathrm{BPb}(\mu \mathrm{g} / \mathrm{d} l)$ & 17.1 & $2.1-62.9$ \\
$\mathrm{ALA}-\mathrm{P}(\mu \mathrm{g} / l)$ & 11.8 & $6.4-65.5$ \\
$\mathrm{ALA}-\mathrm{B}(\mu \mathrm{g} / l)$ & 6.6 & $3.1-38.7$ \\
$\mathrm{ALA}-\mathrm{U}(\mathrm{mg} / \mathrm{g}$ Cre $)$ & 0.81 & $0.22-3.97$ \\
$\mathrm{ALAD}(\mathrm{u}: \mu \mathrm{mol}$ ALA $/ \mathrm{min} / \mathrm{l} \mathrm{RBC})$ & 42.4 & $5.2-81.8$ \\
\hline
\end{tabular}

*Minimum and maximum.

Table 2. Pearson's product-moment correlation coefficients ( $\mathrm{r}$ ) between blood lead $(\mathrm{BPb})$ and $\delta$-aminolevulinic acid (ALA) levels, and log-transformed $\delta$-aminolevulinic acid (ALAD) activity in 186 lead workers

\begin{tabular}{lcc}
\hline & \multicolumn{2}{c}{ Correlation coefficient } \\
\cline { 2 - 3 } & $\mathrm{N}=186$ & $\mathrm{~N}=154^{\mathrm{a}}$ \\
\hline ALA in plasma & $0.748^{*}$ & $0.793^{*}$ \\
ALA in blood & $0.743^{*}$ & $0.756^{*}$ \\
ALA in urine & $0.661^{*}$ & $0.458^{*}$ \\
log-transformed ALAD & $-0.908^{*}$ & $-0.844^{*}$ \\
\hline
\end{tabular}

a: Workers with BPb levels of 2.1-39.4 $\mu \mathrm{g} / \mathrm{d} l . *$ *: $\mathrm{p}<0.001$. 


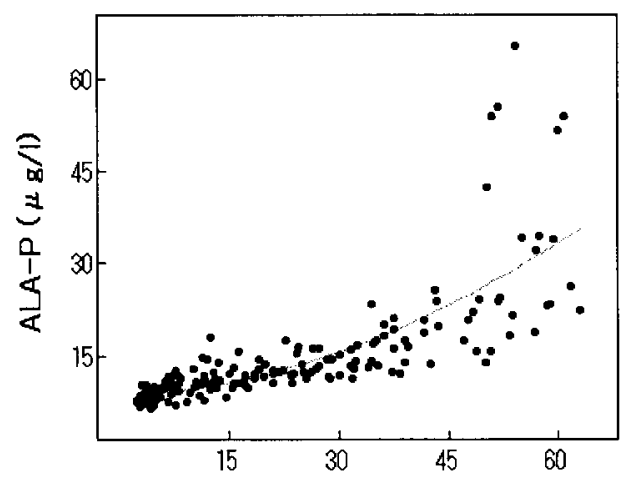

Blood lead concentration ( $\mu \mathrm{g} / \mathrm{dl}$ )

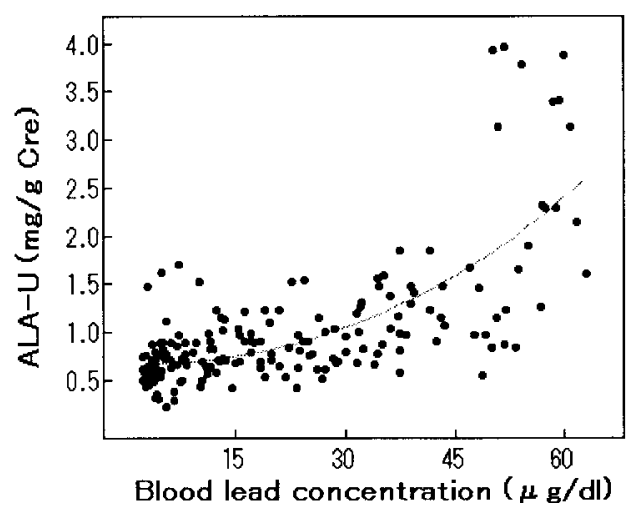

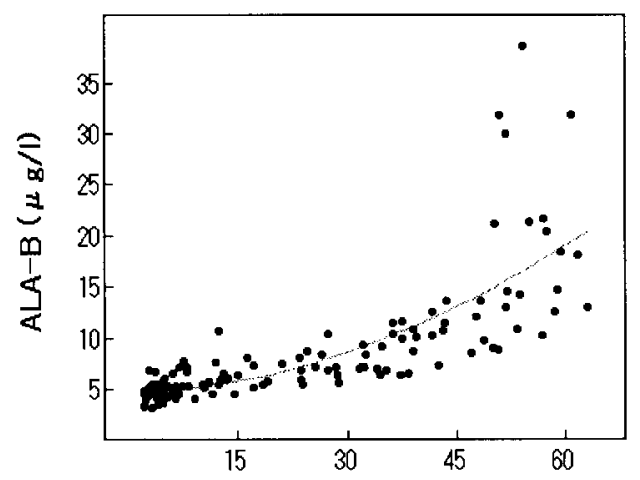

Blood lead concentration $(\mu \mathrm{g} / \mathrm{dl})$

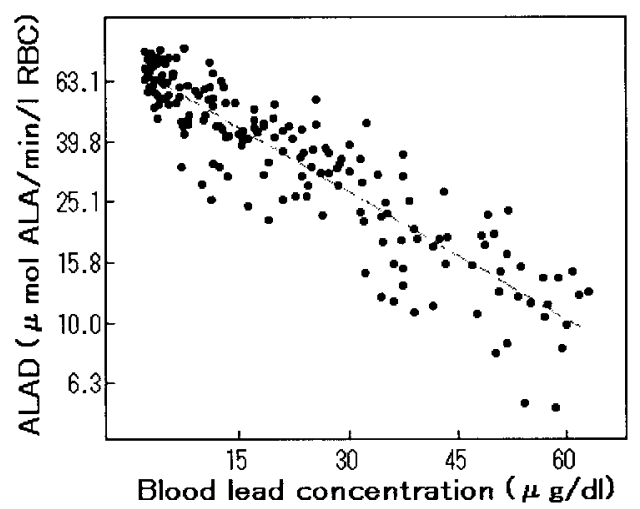

Fig. 2. Dose-effect relations of the blood lead concentration to $\delta$-aminolevulinic acid levels in plasma (ALA-P), blood (ALA-B), and urine (ALA-U) and $\delta$-aminolevulinic acid dehydratase (ALAD) activity in 186 lead workers for benchmark dose (BMD) calculation.

Table 3. Benchmark doses (BMD, $\mu \mathrm{g} / \mathrm{d} l$ ) of blood lead (BPb) and the lower $95 \%$ confidence limits (BMDL, $\mu \mathrm{g} / \mathrm{d} l$ ), set at the $\mathrm{P}_{0}$ of $5 \%$ and $\mathrm{BMR}$ of $5 \%$ after controlling for age, for $\delta$-aminolevulinic acid levels in plasma (ALA-P), blood (ALA-B), and urine (ALA-U), and log-transformed $\delta$-aminolevulinic acid dehydratase (ALAD) activity in two groups (Group A: 186 lead workers with BPb levels of 2.1-62.9 $\mu \mathrm{g} / \mathrm{d}$; Group B: 154 lead workers with $\mathrm{BPb}$ levels of 2.1-39.4 $\mu \mathrm{g} / \mathrm{d} l$ of Group A) ${ }^{\mathrm{a}}$

\begin{tabular}{lccccccc}
\hline & \multicolumn{3}{c}{ Group A } & & \multicolumn{3}{c}{ Group B } \\
\cline { 2 - 3 } & Cutoff* & BMD & BMDL & & Cutoff* & BMD & BMDL \\
\hline ALA-P $(\mu \mathrm{g} / l)$ & 17.9 & 15.3 & 14.3 & & 11.3 & 3.3 & 2.9 \\
ALA-B $(\mu \mathrm{g} / l)$ & 10.7 & 16.6 & 15.3 & & 6.6 & 4.2 & 3.5 \\
ALA-U $(\mathrm{mg} / \mathrm{g}$ Cre) & 1.43 & 20.9 & 19.6 & & 1.1 & 8.8 & 6.8 \\
log-transformed ALAD $(\mathrm{u})$ & 46.9 & 2.9 & 2.6 & & 48.8 & 2.7 & 2.3 \\
\hline
\end{tabular}

a: $\mathrm{P}_{0}$ and $\mathrm{BMR}$ indicate an abnormal probability in unexposed workers and an excess risk in exposed workers, respectively (see Methods). *: Cutoff value, specified by the BMD method, of each of the ALA-related parameters.

probably due to the increased activity of the ALA synthase ${ }^{2,4,16,23,24)}$; consequently, distributions of the ALA were scattered at $\mathrm{BPb}$ levels above $40 \mu \mathrm{g} / \mathrm{d} l$ (Fig. 2). For that reason, we should discuss mainly the outcomes from the 154 lead workers with $\mathrm{BPb}$ levels below $40 \mu \mathrm{g} / \mathrm{d} l$, while consideration for such an inadequate population could occasionally be disregarded.

In the lead workers with BPb levels below $40 \mu \mathrm{g} / \mathrm{d} l$, the BMDs for ALA levels were estimated to be between 3.3 and $8.8 \mu \mathrm{g} / \mathrm{d} l$ (Table 3 ). These values seem to be considerably lower than those recognized in previous studies $^{3-6)}$, and the International Programme on Chemical 
Safety ${ }^{1)}$ has also mentioned that the BPb levels, above which effects are demonstrable with current techniques for parameters that may have clinical significance, are all greater than $20 \mu \mathrm{g} / \mathrm{d} l$. Nevertheless, since recent concern is directed to lead at "subclinical" low levels of exposure ${ }^{15)}$, especially to lead affecting susceptible subpopulations such as young children, the range of $\mathrm{BPb}$ available for the study design should be considered in a future study. But, such a study would make no sense unless the previously estimated threshold were included within the $\mathrm{BPb}$ range.

The BMD for log-transformed ALAD was 2.7 or 2.9 $\mu \mathrm{g} / \mathrm{d} l$ (Table 3 ), implying that the ALAD activity changes almost parallel to the $\mathrm{BPb}$ level. This finding agrees with other studies in the general population, which have confirmed the correlation and the apparent lack of a threshold for inhibition of ALAD in different age groups ${ }^{6,25)}$. The BMDs for ALA-P and ALA-B also followed that for log-transformed ALAD, and the BMD for ALA-U was the highest among ALA-related parameters; the latter finding may have been attributable to the fact that ALA-U is readily affected by urine volume $^{7)}$. The order of these BMDs is biochemically reasonable, and it is accordingly suggested that BMD calculations are biologically plausible, at least at low $\operatorname{doses}^{10,13)}$.

In conclusion, the inhibition of ALAD due to lead at low levels of less than $10 \mu \mathrm{g} / \mathrm{d} l$ is suggested to cause immediately increased levels of ALA-P and ALA-B. Although such subtle changes in ALA at low levels of exposure may hardly lead to direct impairment or disability in human life, this conclusion provides a notion of the discernible threshold of lead. On the other hand, the odds ratio, relative risk and the $95 \%$ confidence intervals have been frequently used in many epidemiological studies. These values imply only the strength of associations, but not a benchmark for preventive goals. For the development of effective public health policy, therefore, further research is necessary to identify the threshold, as noted above, of $\mathrm{BPb}$ affecting different target organs such as the brain, peripheral nerves and kidneys, possibly by using the same method.

Acknowledgments: We thank to Prof. Philippe Grandjean and Dr. Esben Budtz-Jørgensen for their valuable advice on BMD calculations. This research was supported by grants-in-aid for scientific research from the Nissan Science Foundation and the Japan Ministry of Education, Culture, Science and Technology.

\section{References}

1) International Programme on Chemical Safety. Environmental Health Criteria 165: Inorganic Lead. Geneva: World Health Organization, 1995

2) T Sakai: Biomarkers of lead exposure. Ind Health 38,
127-142 (2000)

3) S Selander and K Cramer: Interrelationships between lead in blood, lead in urine and ALA in urine during lead work. Br J Ind Med 27, 28-39 (1970)

4) PA Meredith, MR Moore, BC Campbell, GG Thompson and A Goldberg: Delta-aminolevulinic acid metabolism in normal and lead-exposed humans. Toxicology 9, 1-9 (1978)

5) R Lauwerys, JP Buchet, HA Roels and DD Materne: Relationship between urinary $\delta$-aminolevulinic acid excretion and the inhibition or red cell $\delta$ aminolevulinate dehydratase by lead. Clin Toxicol 7 , 383-388 (1974)

6) HA Roels and R Lauwerys: Evaluation of dose-effect and dose-response relationships for lead exposure in different Belgian population groups (foetus, child, adult men and women). Trace Elem Med 4, 80-87 (1987)

7) K Higashikawa, K Furuki, S Takada, S Okamoto, $H$ Ukai, T Yuasa and M Ikeda: Blood lead level to induce significant increase in urinary $\delta$-aminolevulinic acid level among lead-exposed workers: a statistical approach. Ind Health 38, 181-188 (2000)

8) National Research Council. Toxicological Effects of Methylmercury. Washington DC: National Academy Press, 2000

9) K Crump: Calculation of benchmark doses from continuous data. Risk Anal 15, 79-89 (1995)

10) K Crump: Critical issues in benchmark calculations from continuous data. Crit Rev Toxicol 32, 133-153 (2002)

11) KS Crump, C Van Landingham, C Shamlaye, C Cox, PW Davidson, GJ Myers and TW Clarkson: Benchmark concentrations for methylmercury obtained from the Seychelles Child Development Study. Environ Health Perspect 108, 257-263 (2000)

12) KS Crump, T Kjellström, AM Shipp, A Silvers and A Stewart: Influence of prenatal mercury exposure upon scholastic and psychological test performance: benchmark analysis of a New Zealand Cohort. Risk Anal 18, 701-713 (1998)

13) E Budtz-Jørgensen, N Keiding and P Grandjean: Benchmark dose calculation from epidemiological data. Biometrics 57, 698-706 (2001)

14) K Murata, E Budtz-Jørgensen and P Grandjean: Benchmark dose calculations for methylmercuryassociated delays on evoked potential latencies in two cohorts of children. Risk Anal 22, 465-474 (2002)

15) EK Silbergeld: Lead poisoning: the implications of current biomedical knowledge for public policy. Md Med J 45, 209-217 (1996)

16) T Sakai and Y Morita: $\delta$-Aminolevulinic acid in plasma or whole blood as a sensitive indicator of lead effects, and its relation to the other heme-related parameters. Int Arch Occup Environ Health 68, 126-132 (1996)

17) Y Morita, T Sakai, S Araki, T Araki and Y Masuyama: Usefulness of $\delta$-aminolevulinic acid in blood as an indicator of lead exposure. Jpn J Ind Health 35, 112118 (1993)

18) Y Morita, S Araki, T Sakai, T Araki and Y Masuyama: Determination of delta-aminolevulinic acid in plasma using high-performance liquid chromatography: a 
sensitive indicator of lead effects. Ind Health 32, 8596 (1994)

19) A Okayama, S Fujii and R Miura: Optimized fluorometric determination of urinary $\delta$-aminolevulinic acid by using precolumn derivatization, and identification of the derivative. Clin Chem 36, 1494 1497 (1990)

20) T Sakai, S Yanagihara and K Ushio: Restoration of lead-inhibited $\delta$-aminolevulinate dehydratase activity in whole blood by heat, zinc ion, and (or) dithiothreitol. Clin Chem 26, 625-628 (1980)

21) Murata K, Yano E. Medical Statistics for EvidenceBased Medicine with SPBS User's Guide. Tokyo: Nankodo Publisher, 2002

22) Y Morita, T Sakai, T Araki, K Suzuki, K Oda, S Araki and Y Masuyama: A reference value for deltaaminolevulinic acid in plasma in the population occupationally unexposed to lead. Ind Health $34,57-$ 60 (1996)

23) T Sakai, Y Morita, T Araki, M Kano and T Yoshida: Relationship between delta-aminolevulinic acid dehydratase genotypes and heme precursors in lead workers. Am J Ind Med 38, 355-360 (2000)

24) MR Moore, A Goldberg and AAC Young-Laiwah: Lead effects on the haem-biosynthetic pathway: relationship to toxicity. Ann NY Acad Sci 514, 191-203 (1987)

25) JJ Chisolm, DJ Thomas and TG Hamill: Erythrocyte porphobilinogen synthase activity as an indicator of lead exposure to children. Clin Chem 31, 601-605 (1985) 